The most common diagnoses were: Normal (31\%), Hiatus Hernia (27\%), Non-erosive Gastritis (17\%), Reflux Oesophagitis (11\%). Other frequent diagnoses were: Barrett's oesophagus (7\%), Erosive Gastritis (6\%), Gastric Polyp(s) (5\%), Non-erosive Duodenitis (5\%), Oesophageal Varices (2\%), Erosive Duodenitis (2\%), Duodenal Ulcer (2\%), Gastric Ulcer (2\%). 'Other' was included in the diagnosis field in $18 \%$ of OGDs.

(NB: Multiple indications and diagnoses can be entered for a procedure, hence sum of percentages is greater than $100 \%$ for these categories)

Conclusion The majority of procedures were performed in the $\geq 50$ age group, peaking between ages 70 to 79 , although a quarter of all procedures were performed in people younger than 50. Close to half of OGDs are performed without sedation.

Procedure uploads to the NED continue to increase exponentially year on year. The volume of data and high proportion of sites uploading allows unparalleled insights into OGD practice in the UK.

\section{P13 UNCOVERING THE ENDOSCOPIC PORTAL HYPERTENSION BURDEN IN SIERRA LEONE - AND STARTING TO TREAT IT}

\begin{abstract}
${ }^{1}$ Sorie Conteh*, ${ }^{1}$ Donna Es'e Thomas-Macauley, ${ }^{1}$ Finda Ngongo, ${ }^{2}$ Jamie Catlow, ${ }^{2}$ Chris Wells, ${ }^{2}$ John Hancock, ${ }^{3}$ Chris Mountford, ${ }^{3}$ David Nylander, ${ }^{2}$ Roisin Bevan. ${ }^{1}$ Choithram Memorial Hospital, Freetown, Sierra Leone; ${ }^{2}$ North Tees And Hartlepool NHS Foundation Trust, Stockton-on-Tees, UK; ${ }^{3}$ Newcastle upon Tyne Hospitals NHS Foundation Trust, Newcastle upon Tyne, UK
\end{abstract}

\subsection{6/gutjnl-2020-bsgcampus.88}

Introduction Portal hypertension (PHT) may cause life-threatening bleeding from oesophageal or gastric varices, but if identified can be managed endoscopically or medically. Worldwide, cirrhosis is most commonly due to viral hepatitis. In Sierra Leone, hepatitis B prevalence is estimated at 10\%. Little is known about the rates of cirrhosis or PHT due to a lack of diagnostics. The World Health Organisation (WHO) aims to reduce viral hepatitis mortality by $65 \%$ by the year 2030 in sub-Saharan Africa. Sierra Leone's new endsocopy service can collect data on PHT rates, and through face-to-face and remote training can offer endsocopic mangement of varices. Here we describe the rate of PHT, and use a case report to demostrate impact.

Methods The database of endoscopies performed was interrogated, from the inception of the service in 2016 to November 2019. Cases with PHT were identified, and where possible, the cause of PHT sought. Details of endoscopic therapies were recorded. A case report of one subject was recorded.
Results

- 448 procedures, 55\% male, median age 44 (12-98)

- 35 had PHT changes, 86\% male, median age 47 (25-75)

- 20 cases had oesophageal varices, 7 had gastric varices, 8 had both

- 12 with stigmata of recent bleeding, 1 actively bleeding

- Banding has been performed 9 times ( 2 by visiting UK team, 7 by SL team)

- Hepatitis B was the most frequent cause of liver disease

Case Report Pt A (31M) has been admitted to the government hospital on 4 occasions over 2 years with circulatory collapse and evidence of GI bleeding. Blood transfusions were required ( $\mathrm{Hb}$ level 45). Endoscopy was never offered, but after promotion of the endoscopy service he was referred to Choithram hospital. He underwent OGD with band ligation, and recommendation for further treatment made. His hepatitis B status was established ( $\mathrm{HbsAg}+\mathrm{ve})$. Endoscopic identification and treatment of his PHT will reduce his morbidity, his need for future admissions, and hopefully allow him to return to work.

Conclusions Portal hypertensive changes are frequently identified at endoscopy. Therapy to varices, or recommendations for medical treatment of PHT will reduce the morbidity associated with cirrhosis. As sub-Saharan Africa attempts to reduce the impact of viral hepatitis, the Sierra Leone endoscopy team will be able to play a part by identifiying and treating the PHT complications arising from cirrhotic liver disease. We demonstrate that the skills required for this can be taught in a resource-poor environment.

\section{REFERENCE}

1. Hepatitis B in sub-Saharan Africa: strategies to achieve the 2030 elimination targets. CW Spearman, et al. Lancet Gastroenterol Hepatol 2017; 2:900-909

\section{P14 INITIAL UK EXPERIENCE IN USE OF THE GASTRODUODENAL FULL THICKNESS RESECTION DEVICE}

${ }^{1}$ Phil Boger*, ${ }^{2}$ Bu'Hussain Hayee, ${ }^{2}$ Shraddha Gulati, ${ }^{2}$ Mehul Patel, ${ }^{2}$ Amyn Haji, ${ }^{1}$ Praful Patel, 'Imdadur Rahman. 'Southampton Interventional Endoscopy Unit, University Hospital Southampton, Southampton, UK; ${ }^{2}$ Kings Health Partners Institute of Therapeutic Endoscopy (KITE), London, UK

\subsection{6/gutjnl-2020-bsgcampus.89}

Introduction The gastroduodenal full thickness resection device $\left(\right.$ FTRD $\left.^{\oplus}\right)$ is a new device that allows resection of tethered epithelial or subepithelial lesions (SELs) in the stomach and duodenum, but data on outcomes are limited ${ }^{1}$. Here we present first UK experience of this technique, including technical feasibility, safety and early outcomes.

\begin{tabular}{|c|c|c|c|c|c|c|c|c|}
\hline Case & Age & Sex & Indication & Location & Lesion size & Resection size & RO & Histology \\
\hline 1 & 60 & Male & SEL & Antrum & 24 & 29 & Yes & Fibroid polyp \\
\hline 2 & 66 & Male & SEL & Fundus & 10 & 12 & Yes & Grade 1 neuroendocrine tumour (NET) \\
\hline 3 & 80 & Female & SEL & Duodenum (first part) & 10 & 12 & Yes & Grade 1 NET \\
\hline 4 & 64 & Female & SEL & Duodenum (first part) & 12 & 18 & No & Grade 1 NET \\
\hline 5 & 68 & Female & Suspected non-lifting recurrent adenoma & Duodenum (second part) & 24 & 27 & Yes & Pancreatic heterotopia \\
\hline
\end{tabular}

